\title{
Generation of frequency-chirped density modulation electron beam for producing ultrashort $\mathrm{THz}$ radiation pulse
}

\author{
Haoran Zhang®, Wenxing Wang®, Shimin Jiang, Chen Li, Zhigang He®, \\ Qika Jia, Lin Wang, and Duohui He \\ National Synchrotron Radiation Laboratory, University of Science and Technology of China, \\ Hefei, Anhui, 230029, China
}

(Received 8 October 2019; accepted 27 January 2020; published 13 February 2020)

\begin{abstract}
A new scheme is proposed to generate electron beams with a frequency-chirped density modulation for producing ultrashort $\mathrm{THz}$ radiation pulse in an optimally tapered undulator. In this scheme, an energy chirped electron beam is sequentially modulated in two modulators by interacting with two laser pulses with the same central frequency but different chirp rates, practically one laser pulse is linearly chirped and the other is chirpfree. Two dispersion sections are applied to transfer the energy modulation formed in the modulators to the desired density modulation. The central frequency of the density modulation of the electron beam can be continuously tuned by changing the compression coefficients of the dispersion sections, and the chirp rate of the final modulation frequency can also be tuned by either varying the compression coefficients or the linear chirp rates of the modulation laser pulses. The generation of ultrashort and broadband $\mathrm{THz}$ radiation pulses is also briefly discussed.
\end{abstract}

DOI: 10.1103/PhysRevAccelBeams.23.020704

\section{INTRODUCTION}

Terahertz $(\mathrm{THz})$ radiation is finding its use in an increasingly wide variety of applications. Ultrashort flashes of strong electric or magnetic field transients of $\mathrm{THz}$ light have been employed to control and detect materials [1-9].

$\mathrm{THz}$ undulator radiation sources are widely studied in the accelerator community [10-17], and there is continuous interest to produce radiation pulses with shorter duration [18-20]. To meet the demands of ultrashort $\mathrm{THz}$ radiation pulses, a scheme is proposed to generate quasi-halfcycle $\mathrm{THz}$ pulses, which is emitted from a frequencychirped electron bunch train traversing an optimally tapered undulator [21,22].

For generating such an ultrashort and broadband $\mathrm{THz}$ radiation pulse in the tapered undulator, the key is to obtain a frequency-chirped electron bunch train or microbunched beam. With an equally spaced laser pulse train to illuminate the cathode of a photo-cathode rf gun, frequency-chirped electron bunch train can be generated by optimizing the launching rf phase [22]. The laser modulation method is widely used in the FEL [23], and many schemes are proposed to generate equally spaced microbunched beams

\footnotetext{
*hezhg@ustc.edu.cn

Published by the American Physical Society under the terms of the Creative Commons Attribution 4.0 International license. Further distribution of this work must maintain attribution to the author(s) and the published article's title, journal citation, and DOI.
}

for narrow-band $\mathrm{THz}$ radiation [24-26]. A different generation method has also been proposed to modulate the electron bunch for generating a frequency-chirped microbunching in the beam, in which the electron beam interacts with a dual-frequency laser and emits intense coherent radiation at the difference frequency in a tapered undulator [27]. However, an optical parametric amplifier system is required to provide two laser pulses with different central frequencies.

Here, we propose a new scheme with two same centralfrequency laser pulses for modulating the electron bunch to obtain such a desired microbunched beam, leading to the generation of an ultrashort $\mathrm{THz}$ pulse in an optimally tapered undulator. This scheme is similar to that of using two laser pulses with the same frequency to modulate the electron beam for generating an equally spaced microbunched beam [25], but we replace the conventional laser with a frequency-chirped laser. In this way, it can generate frequency-chirped $\mathrm{THz}$ density modulation in a relativistic electron beam.

In this scheme, an energy chirped electron beam is modulated by a laser pulse with a central angular frequency of $\omega_{0}$ and a chirp term of $\alpha_{1}$ in the first modulator. Under the density modulation of the first dispersion section (compression coefficient of $C_{1}$ ), the central angular frequency is compressed to $\omega=C_{1} \omega_{0}$ and the chirp term becomes $\alpha=C_{1}^{2} \alpha_{1}$. Then, the beam is sent into the second modulator and modulated by the other laser pulse with the same central angular frequency of $\omega_{0}$ and the chirp term of $\alpha_{2}$. After the density modulation through the second 
dispersion section (compression coefficient of $C_{2}$ ), the central angular frequency becomes $\omega=C_{1} C_{2} \omega_{0}-C_{2} \omega_{0}$, and the chirp term becomes $\alpha=C_{1}^{2} C_{2}^{2} \alpha_{1}-C_{2}^{2} \alpha_{2}$. It can be seen that by adjusting the compression coefficient of dispersion sections related to the initial energy chirp and dispersion strength, one can change the central frequency and chirp rate of the final density modulation beam.

This paper is organized as follow. In Sec. II, the detailed theoretical analysis of the proposed electron bunch modulation method is discussed. In Sec. III, simulations are performed to verify the feasibility of this scheme and the generation of ultrashort $\mathrm{THz}$ radiation is presented in Sec. IV. Lastly, summary and conclusion are given in Sec. V.

\section{LASER-ELECTRON INTERACTION SCHEME AND MODULATION THEORY}

The layout of the proposed scheme is shown in Fig. 1, which consists of two modulators and two dispersion sections. An energy chirped electron beam passes through the first modulator and is modulated by the laser with central frequency $\omega_{0}$. This frequency will be compressed after the beam passes through the first dispersion section, so that it can generate frequency difference compared with the other laser in the second modulator. By choosing suitable chirp terms of the two lasers $\left(\alpha_{1}\right.$ and $\left.\alpha_{2}\right)$, it can be generated in the final dispersion section that the required frequencychirped density modulation beam.

If the length of the beam is much longer than that of the laser, we can ignore the initial variation of beam current intensity and assume a longitudinal uniform current beam. Considering a Gaussian energy distribution with an energy chirp, the initial distribution function of the longitudinal phase space is expressed as follow:

$$
f_{0}(t, p)=\frac{N_{0} e^{-\frac{(p-h t)^{2}}{2}}}{\sqrt{2 \pi}},
$$

where $N_{0}$ is the number of electrons per unit length of the beam, $p=\left(E-E_{0}\right) / \sigma_{E}$ is the energy deviation, $E_{0}$ is the average beam energy, and $\sigma_{E}$ is the slice energy spread. $h=d p / d t=d E /\left(\sigma_{E} d t\right)$ is the energy chirp rate of the beam and $t$ is the longitudinal coordinate within the beam.

After passing through the first modulator, the beam energy is modulated with the amplitude $\Delta E_{1}$, so that the

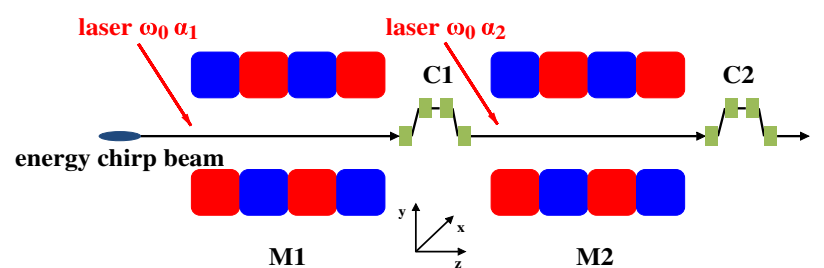

FIG. 1. Schematic layout for the generation of electron beams with a frequency-chirped density modulation.

dimensionless energy deviation is converted from initial $p$ to $p^{\prime}=p+A_{1} e^{-t^{2} /\left(2 \sigma_{t 1}^{2}\right)} \cos \left(\omega_{1} t+\alpha_{1} t^{2}\right)$, where $A_{1}=$ $\Delta E_{1} / \sigma_{E}$ and $\sigma_{t 1}$ is the rms length of laser pulse. The distribution function of the electron beam becomes:

$$
\begin{aligned}
& f_{1}(t, p) \\
& \quad=\frac{N_{0}}{\sqrt{2 \pi}} \exp \left\{-\frac{1}{2}\left[p-A_{1} e^{-\frac{t^{2}}{2 \sigma_{t 1}^{2}}} \cos \left(\omega_{1} t+\alpha_{1} t^{2}\right)-h t\right]^{2}\right\} .
\end{aligned}
$$

Then, the beam enters the first dispersion section with a small momentum compaction $R_{56}^{(1)}$ which changes the longitudinal coordinate $t$ to $t^{\prime}=t+R_{56}^{(1)} \sigma_{E} p /\left(c E_{0}\right)=$ $t+K_{1} p$, where $t \approx z / c$ and $\mathrm{c}$ is the light speed, so the distribution becomes:

$$
\begin{aligned}
f_{2}(t, p)= & \frac{N_{0}}{\sqrt{2 \pi}} \exp \left\{-\frac{1}{2}\left[p-A_{1} e^{-\frac{\left(t-K_{1} p\right)^{2}}{2 \sigma_{t 1}^{2}}} \cos \left[\omega_{1}\left(t-K_{1} p\right)\right.\right.\right. \\
& \left.\left.\left.+\alpha_{1}\left(t-K_{1} p\right)^{2}\right]-h\left(t-K_{1} p\right)\right]^{2}\right\} .
\end{aligned}
$$

Similar to the derivations expressed above, the final distribution function at the exit of the second dispersion section can be easily obtained. The beam energy modulation in the second modulator is $p^{\prime}=p+A_{2} e^{-t^{2} /\left(2 \sigma_{t 2}^{2}\right)}$ $\cos \left(\omega_{2} t+\alpha_{2} t^{2}+\phi\right)$, where $\phi$ is the phase difference between the two lasers and the density modulation in the second dispersion section is $t^{\prime}=t+R_{56}^{(2)} \sigma_{E} p /\left(c E_{0}\right)=$ $t+K_{2} p$. Finally, the distribution function becomes:

$$
\begin{aligned}
f_{f}(t, p)= & \frac{N_{0}}{\sqrt{2 \pi}} \exp \left[-\frac{1}{2}\left(G(p)-A_{1} e^{\frac{-\left[t-K_{2} p-K_{1} G(p)\right]^{2}}{2 \sigma_{t 1}^{2}}} \cos \left\{\omega_{1}\left[t-K_{2} p-K_{1} G(p)\right]\right.\right.\right. \\
& \left.\left.\left.+\alpha_{1}\left[t-K_{2} p-K_{1} G(p)\right]^{2}\right\}-h\left[t-K_{2} p-K_{1} G(p)\right]\right)^{2}\right], \\
G(p)= & p-A_{2} e^{-\frac{\left(t-K_{2} p\right)^{2}}{2 \sigma_{12}^{2}}} \cos \left[\omega_{2}\left(t-K_{2} p\right)+\alpha_{2}\left(t-K_{2} p\right)^{2}+\phi\right] .
\end{aligned}
$$



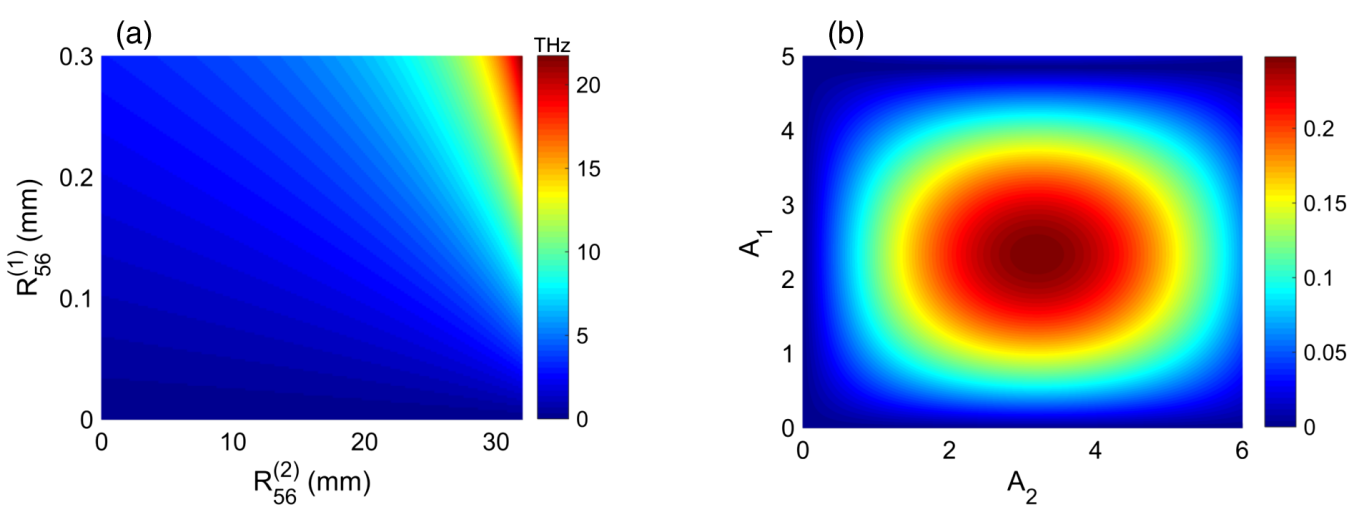

FIG. 2. (a) Central frequency of the density modulated electron beam VS dispersion strengths $R_{56}$; (b) Bunching factor VS laser modulation amplitudes $A$.

The beam with energy chirp $(p=h t)$ will be compressed or stretched by the dispersion section $\left[t^{\prime}=t+K p=\right.$ $(1+h K) t]$. We set the compression factors of the two dispersion sections as $C_{1}=d t / d t^{\prime}=1 /\left(1+h K_{1}\right)$ and $C_{2}=\left(1+h K_{1}\right) /\left[1+h\left(K_{1}+K_{2}\right)\right]$, for the two laser pulses with the same frequency $\omega_{0}=\omega_{1}=\omega_{2}$, the central frequency and chirp term of the final density modulated beam are expressed as follows:

$$
\begin{aligned}
& \omega=\left(C_{1} C_{2}-C_{2}\right) \omega_{0} \\
& \alpha=C_{1}^{2} C_{2}^{2} \alpha_{1}-C_{2}^{2} \alpha_{2} .
\end{aligned}
$$

It is found that the concerned parameters, central frequency and chirp term, are determined by the compression factors when the initial values are set.

We make a $800 \mathrm{~nm}$ laser as seed to modulate the beam with an energy chirp $h=-80 \mathrm{ps}^{-1}$. According to Eq. (5), it is shown in Fig. 2(a) that the variation of central modulation frequency with the dispersion strength $R_{56}$. When $R_{56}^{(1)}=0.264 \mathrm{~mm}$ and $R_{56}^{(2)}=27.4 \mathrm{~mm}$, an approximate central modulation frequency of $10 \mathrm{THz}$ (where $C_{1} C_{2}-C_{2} \approx 0.027$ ) can be reached.

The final density modulation amplitude is relative to the energy modulation amplitudes $A_{1}$ and $A_{2}$. To get an optimal density modulation amplitude, we ignore the chirp term $\left(\alpha_{1}=\alpha_{2}=0\right)$ and use the bunching factor [25] under periodic modulation to represent the density modulation amplitude. The expression of bunching factor here is

$$
\begin{aligned}
b= & \left|J_{-1}\left(-\frac{h A_{2} K_{1} K_{2} \omega_{0}}{1+h B_{1}}\right) J_{1}\left(\frac{A_{1} \omega_{0} B_{1}}{1+h\left(K_{1}+K_{2}\right)}\right)\right| \\
& \times \exp \left\{-\frac{B_{1}^{2}}{2\left[1+h\left(K_{1}+K_{2}\right)\right]^{2}}\right\},
\end{aligned}
$$

and the calculation result is shown in Fig. 2(b). It is easy to find out that when $A_{1}=2.35$ and $A_{2}=3.15$, the bunching factor reaches the maximum value.

The two laser pulses have the same rms pulse width $\sigma_{t}=4 \mathrm{ps}$ and different chirp rates, $\alpha_{1}=1 \mathrm{ps}^{-2}$ and $\alpha_{2}=0$, respectively. By solving Eq. (4) with the above parameters, the detailed evolution of longitudinal phase space is shown in Fig. 3. In the first modulator, the beam is modulated at the angular frequency of $\omega_{0}+2 \alpha_{1} t$ [see Fig. 3(a)], which is compressed to $C_{1} \omega_{0}+2 C_{1}^{2} \alpha_{1} t$ after the first dispersion section. It can be seen in the inset of Fig. 3(b) that the energy modulation in $\mathrm{THz}$ domain appears in the titled phase space which is introduced by the frequency difference of $C_{1} \omega_{0}$ and $\omega_{0}$. Then the phase space is further titled by the second dispersion section [see Fig. 3(c)] to obtain the density modulation at angular (a)

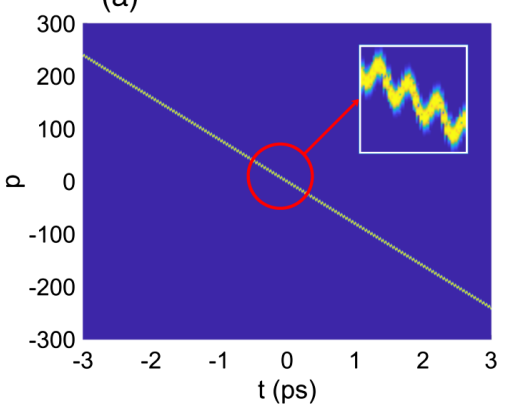

(b)

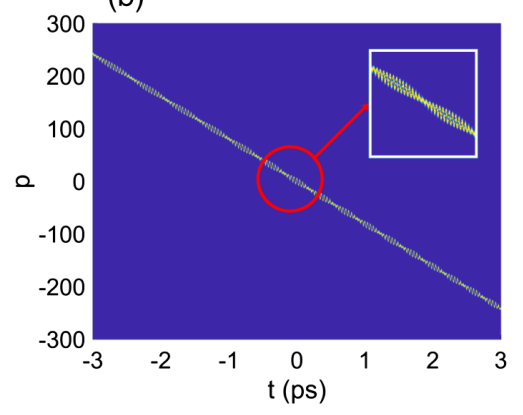

(c)

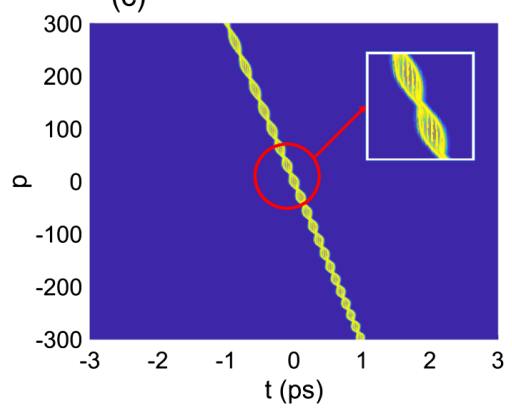

FIG. 3. Phase space evolution of the beam at different positions of (a) the exit of modulator1; (b) the exit of modulator2; (c) the exit of the second dispersion section. The head of the beam is on the right side. 


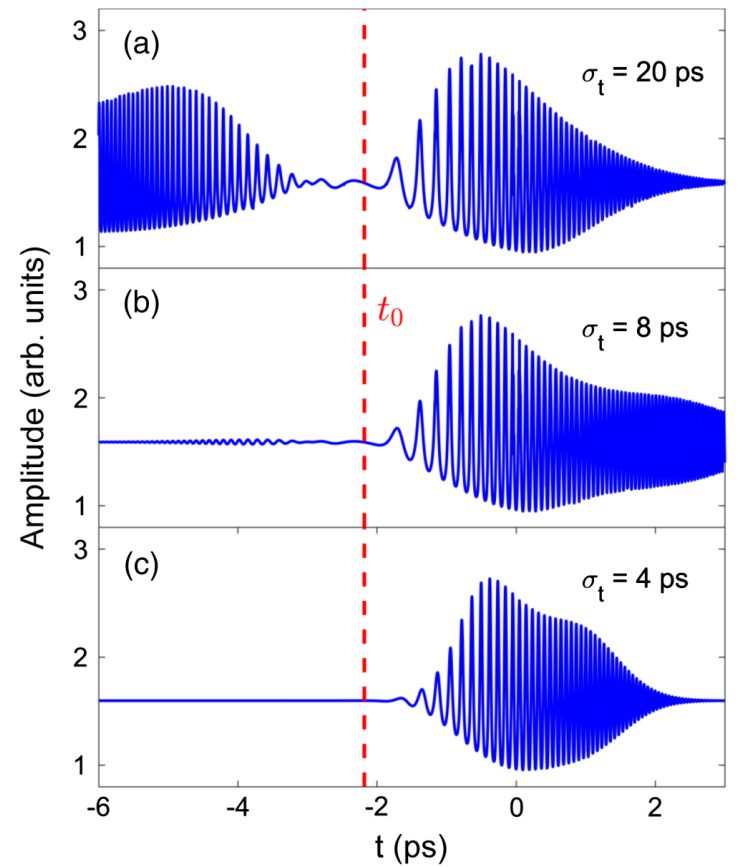

FIG. 4. Final beam density distribution for different laser pulse lengths. (a) $\sigma_{t}=20 \mathrm{ps}$; (b) $\sigma_{t}=8 \mathrm{ps}$; and (c) $\sigma_{t}=4 \mathrm{ps}$. The red dotted line is the symmetrical axis $t_{0}$. The head of the beam is on the right side.

frequency of $(\omega+2 \alpha t)$, and the corresponding density distribution is shown in Fig. 4(c).

Since the modulation, the phase space of electron beam has a form function which is quasiproportional to $\cos \left(\omega t+\alpha t^{2}\right)$ and there is a symmetrical axis of $t_{0}=$ $-\omega / 2 \alpha$. For the above parameters, the axis is $t_{0}=-2.2 \mathrm{ps}$ which is the red dotted line in Fig. 4. As shown in Fig. 4(a), when laser pulses $\sigma_{t}=20 \mathrm{ps}$, there are two macrobunches appearing in the density distribution, which have opposite chirp rates. To get the required microbunches with intervals of monotonous variation, the modulation should not appear in the left side of the axis (or right side when $t_{0}>0$ ). So the relationship between the pulse length $\sigma_{t}$ of the laser and the symmetrical axis should roughly satisfy with $\sigma_{t} / C_{1} C_{2} \leq|\omega / 2 \alpha|$. Then the condition of $\sigma_{t} \leq 8 \mathrm{ps}$ is obtained for the present case, and the density distribution is shown in Fig. 4(b) when $\sigma_{t}=8$ ps.

\section{SIMULATION RESULTS OF ELECTRON BEAM MODULATION}

We develop an one-dimensional code for numerical simulation in order to further explore and verify the feasibility of this scheme.

The basic FEL equations, energy equation, phase equation and wave equation of the light field are solved in the code. The electron beam makes an oscillating motion under the magnetic field of the undulator and exchanges energy according to the phase relation with the external field, losing or gaining energy. The radiation field is slipping one resonance wavelength after the electron beam transiting one undulator period. The equations used in this code are expressed as follow,

$$
\begin{aligned}
{\left[\left(\frac{\partial^{2}}{\partial z^{2}}-\frac{1}{c^{2}} \frac{\partial}{\partial t}\right)\right] \vec{E}_{\perp} } & =\frac{4 \pi}{c^{2}} \frac{\partial \vec{J}_{\perp}}{\partial t} \\
\frac{d \theta}{d z} & =2 k_{u}\left(\frac{\gamma-\gamma_{r}}{\gamma_{r}}\right) \\
\frac{d \gamma}{d z} & =\frac{e}{m_{e} c^{2}} \frac{\beta_{\perp}}{\beta} \vec{E}_{\perp}
\end{aligned}
$$

The radiation field is solved by the first equation of Eq. (7), the wave equation. The diffraction effect in the transverse direction is neglected since the simple physical process during the modulation. The first term on the left of this differential equation is related to the amplification and the second term is related to the slippage. $\vec{E}_{\perp}$ is the electric field of transverse radiation, $\vec{J}_{\perp}=-e c \sum \delta\left(\vec{r}-\vec{r}_{j}(t)\right) \vec{\beta}_{\perp}$ is the transverse current density, $e$ is electron charge, $\vec{r}_{j}$ is the trajectory of the $j_{t h}$ electron and $\vec{\beta}_{\perp}$ is the electron transverse velocity normalized to $c$. In the electron dynamics, only the longitudinal relative position is considered, which comes from the second equation of Eq. (7), phase equation, where $\gamma_{r}$ is the resonant energy. The space charge effect of the electron is also neglected. The electron beam exchanges energy with the external field by the third equation of Eq. (7), energy equation. The beam transverse velocity $\beta_{\perp}$ is introduced by the magnetic field and definitively leads to the energy exchange. The wave equation is firstly solved in the spectral domain and it regards the electron beam calculated from the phase equation and energy equation as source. Then the field is transformed to time domain by inverse Fourier transform to interact with the electron beam. To reach the best precision, the leap frog integration is used to deal with the coupling of field and electron, where the source term is evaluated in the middle of the integration step.

In this numerical simulation, in order to meet the requirements of calculation accuracy, the inner calculation parameters are chosen as follow, the time grid size of electron beam is $0.5 \mathrm{fs}$. The frequency resolution of radiation field is about $0.12 \mathrm{THz}$ and the time resolution is $0.08 \mathrm{fs}$. The longitudinal integration step $d z=\lambda_{u} / 100$, where $\lambda_{u}$ is the undulator period.

The main parameters for simulation are listed in Table I. We consider a conventional $800 \mathrm{~nm}$ Ti: sapphire laser and a moderately $80 \mathrm{MeV}$ relativistic electron beam. The beam has a uniform distribution with an energy chirp rate of $h=-80 \mathrm{ps}^{-1}$, and a slice energy spread of $8 \mathrm{keV}$. The final density modulation amplitude can be maximized when the energy modulation amplitude are $19 \mathrm{keV}$ and $25 \mathrm{keV}$, respectively. Its numerical results are shown in Fig. 5. 
TABLE I. Main parameters for simulation.

\begin{tabular}{lc}
\hline \hline Parameter & Value \\
\hline Electron beam energy & $80 \mathrm{MeV}$ \\
Slice energy spread & $8 \mathrm{keV}$ \\
Laser wavelength in M1 and M2 & $800 \mathrm{~nm}$ \\
Laser liner chirp $\alpha$ & $1 \mathrm{ps}^{-2}$ \\
Period of M1 and M2 & $3 \mathrm{~cm}$ \\
Number of periods for M1 and M2 & 6 \\
Energy modulation amplitude in M1 & $19 \mathrm{keV}$ \\
Energy modulation amplitude in M2 & $25 \mathrm{keV}$ \\
$R_{56}$ for C1 & $0.26 \mathrm{~mm}$ \\
$R_{56}$ for C2 & $27.4 \mathrm{~mm}$ \\
\hline \hline
\end{tabular}

At the exit of second dispersion section, there is a frequency-chirped $\mathrm{THz}$ envelope appearing in the longitudinal phase space [see Fig. 5(a)] and the current peaks are generated after the density modulation [see Fig. 5(b)]. It is apparent that the intervals between the adjacent peaks are decreasing along the motion direction of the electron beam.

\section{GENERATION OF ULTRASHORT AND BROADBAND THZ RADIATION}

The above code can also be used to simulate the radiation process in a specific tapered undulator. It is necessary to investigate the diffraction effect, so the diffraction term $\nabla_{\perp}^{2}$ is added to the wave equation. The results include radiation field in both time and frequency domain, which is checked with the code in Ref. [21]. We use the frequency-chirped density modulation electron beam obtained from the above section as the initial distribution, and the time grid size of electron beam is set at $10 \mathrm{fs}$ here which is enough to ensure the computation precision.

The magnetic field intensity of the tapered undulator is optimized by making the resonant wavelengths of each period equal to the intervals between adjacent microbunches along the beam direction, leading to the generation

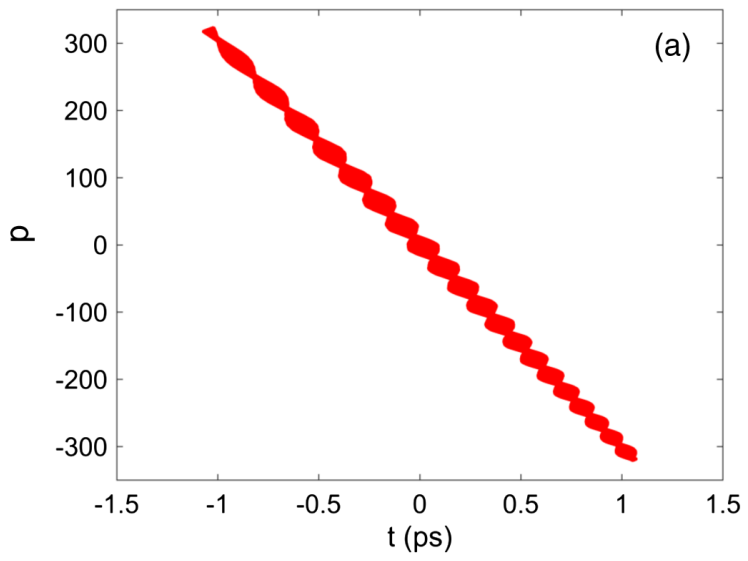

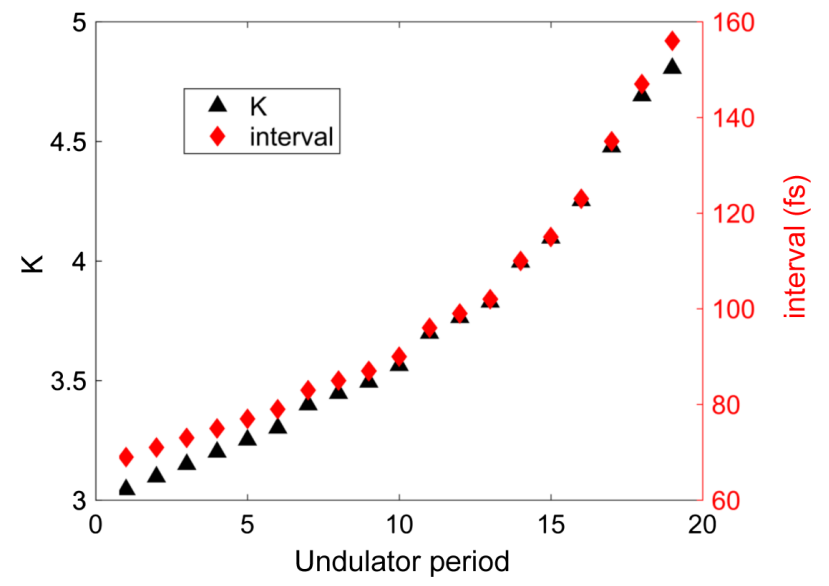

FIG. 6. $K$ value of the $n_{t h}$ period of the tapered undulator, which is calculated based on the intervals between adjacent microbunches.

of an ultrashort radiation pulse [20,21]. We choose 20 microbunches with approximate consistent current peaks, and a tapered undulator with corresponding 19 periods is used. The magnetic field intensity increases along the undulator while the intervals between microbunches increase from beam head to tail (see Fig. 6).

With the undulator period length $\lambda_{u}=18 \mathrm{~cm}$, the rms radial beam size $\sigma_{r}=1 \mathrm{~mm}$ and the total bunch charge $Q=1 \mathrm{nC}$, the simulation results at the exit of the tapered undulator are illustrated in Fig. 7. The final radiation pulse includes one main peak with some low intensity sidebands. The on-axis peak electric field reaches about $40 \mathrm{MV} / \mathrm{m}$ and its spectrum covers from $5 \mathrm{THz}$ to $15 \mathrm{THz}$, which agrees with $\omega+2 \alpha t \simeq(10 \pm 5) \mathrm{THz}$. Although the center peak electric field is quasi-one-cycle, there are always some sidebands around it because of the finite numbers of microbunches. The value of the peak electric field depends on many factors except for the bunch charge and the pulse width of microbunches.

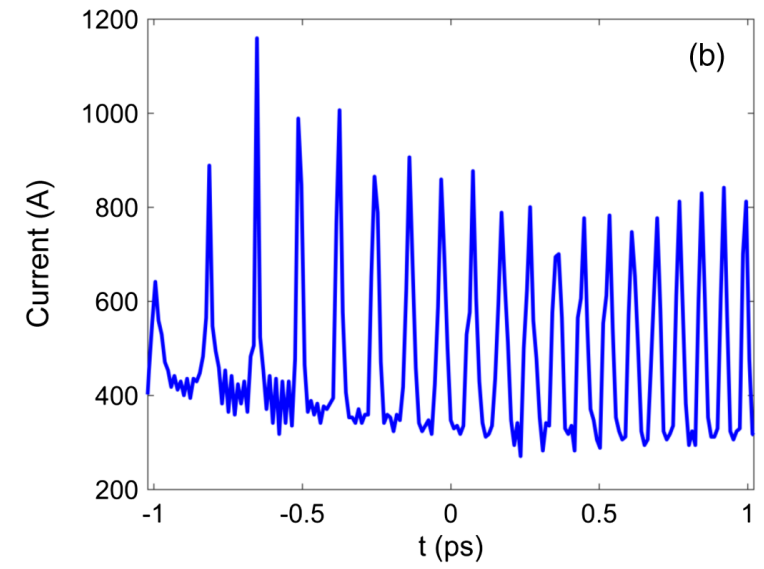

FIG. 5. Simulation results of the modulation at the exit of the second dispersion section. (a) Longitudinal phase space; (b) density distribution. The head of the beam is on the right side. 

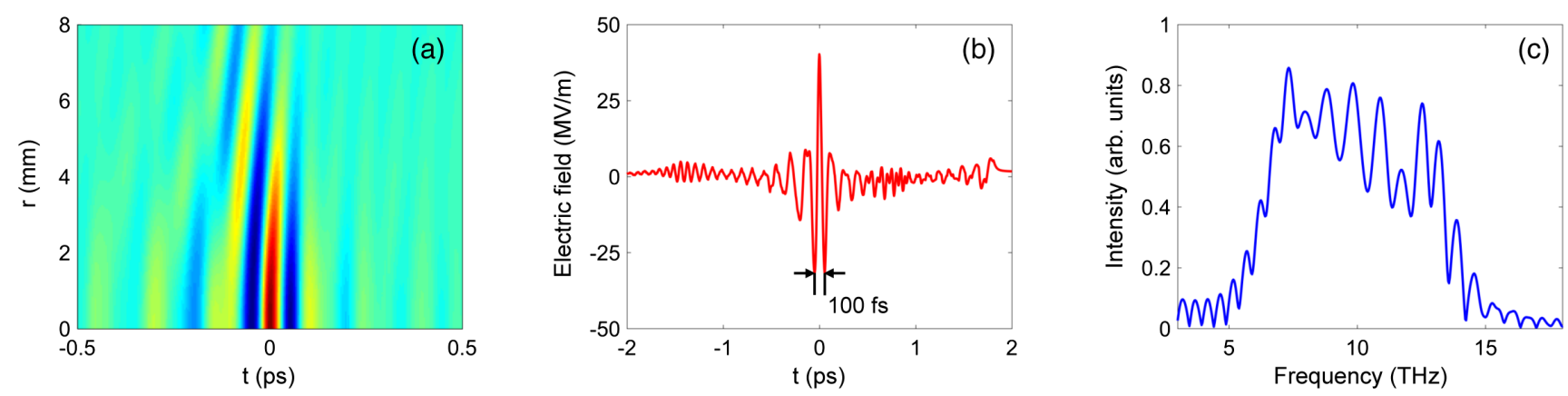

FIG. 7. (a) THz radiation distribution in time-space domain as function of the time and radial position. (b) On-axis electric field in the time domain and (c) the corresponding radiation spectrum. The wavefront is on the right side.

When optimize taper undulator, the constant energy $80 \mathrm{MeV}$ is used in the resonance condition. The maximal frequency deviation caused by the energy difference between the adjacent microbunches is $0.015 \mathrm{THz}$, which is far less than the minimal frequency difference of $0.4 \mathrm{THz}$ between the adjacent undulator periods. Therefore, the effect of energy chirp on the peak electric field can be neglected. The radial beam size $\sigma_{r}$ is a critical parameter to

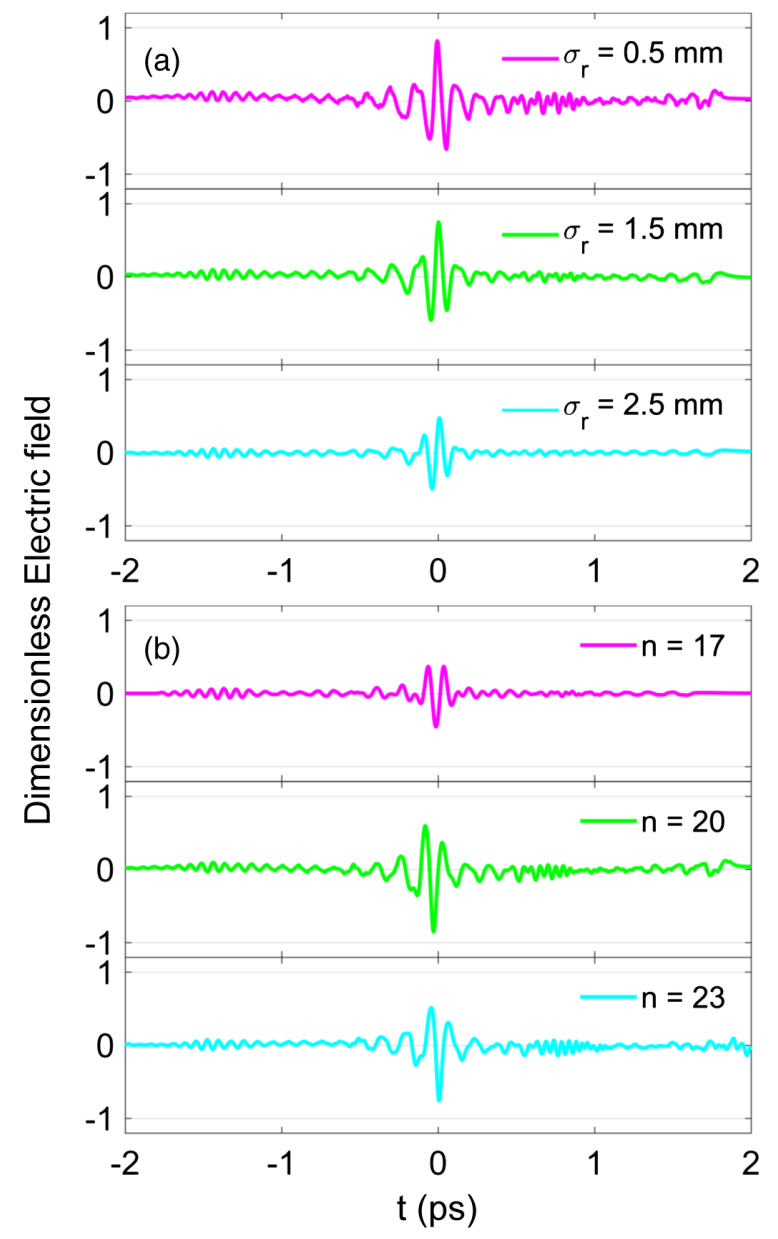

FIG. 8. On-axis electric field with (a) the radial beam size and (b) the microbunch numbers. affect the field intensity, the peak electric field and the intensity of sidebands decrease with the increase of the radial beam size (see Fig. 8). The on-axis electric field with the different numbers of microbunches is shown in Fig. 8(b). As the increasing of the microbunch number from 17 to 20 , the intensity of central peak field is increasing since more electric field components are superimposed on a fixed phase. When the number of microbunches increase to 23 , the peak field does not continuous increase. Because a longer undulator can cause more serious diffraction effect.

\section{CONCLUSION}

This paper mainly present and analyze a strategy to generate electron beams with a frequency-chirped density modulation which can satisfy the requirement of generation of ultrashort $\mathrm{THz}$ radiation from a optimally tapered undulator. This method can impose a $\mathrm{THz}$ modulation in the energy chirped electron beam, which is accomplished by two seed laser pulses with the same center frequency, and one of them is introduced a liner chirp, the other is chirpfree. The final central modulation frequency and the chirp term can be varied by changing the dispersion strengths of dispersion sections and the initial energy chirp. It can avoid the complex optical parametric amplifier system and modify the final distribution easily. Using the frequency-chirped density modulation beam, the ultrashort and broadband $\mathrm{THz}$ radiation can be generated.

\section{ACKNOWLEDGMENTS}

We would like to acknowledge Dr. V. A. Goryashko for sharing the simulation code of $\mathrm{THz}$ radiation calculation. This work is supported by National Natural Science Foundation of China (NSFC) under Grants No. 11775216 and No. 51627901.

[1] M. Liu, H. Y. Hwang, H. Tao, A. C. Strikwerda, K. Fan, G. R. Keiser, A. J. Sternbach, K. G. West, S. Kittiwatanakul, J. Lu et al., Terahertz-field-induced insulator-to-metal 
transition in vanadium dioxide metamaterial, Nature (London) 487, 345 (2012).

[2] T. L. Cocker, D. Peller, P. Yu, J. Repp, and R. Huber, Tracking the ultrafast motion of a single molecule by femtosecond orbital imaging, Nature (London) 539, 263 (2016).

[3] X. Li, M. Bamba, N. Yuan, Q. Zhang, Y. Zhao, M. Xiang, K. Xu, Z. Jin, W. Ren, G. Ma, S. Cao, D. Turchinovich, and J. Kono, Observation of Dicke cooperativity in magnetic interactions, Science 361, 794 (2018).

[4] E. J. Sie, C. M. Nyby, C. Pemmaraju, S. J. Park, X. Shen, J. Yang, M. C. Hoffmann, B. Ofori-Okai, R. Li, A. H. Reid et al., An ultrafast symmetry switch in a Weyl semimetal, Nature (London) 565, 61 (2019).

[5] T. Kubacka, J. A. Johnson, M. C. Hoffmann, C. Vicario, S. De Jong, P. Beaud, S. Grübel, S.-W. Huang, L. Huber, L. Patthey et al., Large-amplitude spin dynamics driven by a $\mathrm{THz}$ pulse in resonance with an electromagnon, Science 343, 1333 (2014).

[6] B. Zaks, R.-B. Liu, and M.S. Sherwin, Experimental observation of electron-hole recollisions, Nature (London) 483, 580 (2012).

[7] M. Tonouchi, Cutting-edge terahertz technology, Nat. Photonics 1, 97 (2007).

[8] T. Kampfrath, K. Tanaka, and K. A. Nelson, Resonant and nonresonant control over matter and light by intense terahertz transients, Nat. Photonics 7, 680 (2013).

[9] P. Salén, M. Basini, S. Bonetti, J. Hebling, M. Krasilnikov, A. Y. Nikitin, G. Shamuilov, Z. Tibai, V. Zhaunerchyk, and V. Goryashko, Matter manipulation with extreme terahertz light: Progress in the enabling THz technology, Phys. Rep. 836, 1 (2019).

[10] Y.-C. Huang, Desktop megawatt superradiant free-electron laser at terahertz frequencies, Appl. Phys. Lett. 96, 231503 (2010).

[11] B. Green, S. Kovalev, V. Asgekar, G. Geloni, U. Lehnert, T. Golz, M. Kuntzsch, C. Bauer, J. Hauser, J. Voigtlaender et al., High-Field High-Repetition-Rate Sources for the Coherent THz Control of Matter, Sci. Rep. 6, 22256 (2016).

[12] X. Su, D. Wang, Q. Tian, Y. Liang, L. Niu, L. Yan, Y. Du, W. Huang, and C. Tang, Widely tunable narrow-band coherent Terahertz radiation from an undulator at THU, J. Instrum. 13, C01020 (2018).

[13] J. Zhang, H. Deng, X. Lin, D. Dai, Q. Sun, S. Lu, T. Yu, H. Zhao, H. Yang, and Z. Dai, Tunable few-cycle coherent terahertz radiation with watt-level power from relativistic femtosecond electron beam, Nucl. Instrum. Methods Phys. Res., Sect. A 693, 23 (2012).
[14] X. Wen, S. Huang, L. Lin, F. Wang, F. Zhu, L. Feng, L. Yang, Z. Wang, P. Fan, J. Hao et al., Superradiant THz undulator radiation source based on a superconducting photo-injector, Nucl. Instrum. Methods Phys. Res., Sect. A 820, 75 (2016).

[15] N. Sei, H. Zen, and H. Ohgaki, Development of intense terahertz coherent synchrotron radiation at KUFEL, Nucl. Instrum. Methods Phys. Res., Sect. A 832, 208 (2016).

[16] H. Li, Y. Lu, Z. He, Q. Jia, and L. Wang, Generation of intense narrow-band tunable terahertz radiation from highly bunched electron pulse train, J. Infrared, Millimeter, and Terahertz Waves 37, 649 (2016).

[17] N. Stojanovic and M. Drescher, Accelerator- and laserbased sources of high-field terahertz pulses, J. Phys. B 46, 192001 (2013).

[18] A. Mak, G. Shamuilov, P. Salén, D. Dunning, J. Hebling, Y. Kida, R. Kinjo, B. McNeil, T. Tanaka, N. Thompson et al., Attosecond single-cycle undulator light: A review, Rep. Progr. Phys. 82, 2 (2018).

[19] Y. Kida, R. Kinjo, and T. Tanaka, Synthesizing high-order harmonics to generate a sub-cycle pulse in free-electron lasers, Appl. Phys. Lett. 109, 151107 (2016).

[20] T. Tanaka, Proposal to Generate an Isolated Monocycle X-Ray Pulse by Counteracting the Slippage Effect in FreeElectron Lasers, Phys. Rev. Lett. 114, 044801 (2015).

[21] V. A. Goryashko, Quasi-half-cycle pulses of light from a tapered undulator, Phys. Rev. Accel. Beams 20, 080703 (2017).

[22] Z. Ma, Z. Wang, F. Fu, R. Wang, and D. Xiang, Generating quasi-single-cycle $\mathrm{THz}$ pulse from frequency-chirped electron bunch train and a tapered undulator, High Power Laser Sci. Eng. 4, e1 (2016).

[23] E. Hemsing, G. Stupakov, D. Xiang, and A. Zholents, Beam by design: Laser manipulation of electrons in modern accelerators, Rev. Mod. Phys. 86, 897 (2014).

[24] D. Xiang and G. Stupakov, Enhanced tunable narrow-band $\mathrm{THz}$ emission from laser-modulated electron beams, Phys. Rev. ST Accel. Beams 12, 080701 (2009).

[25] Z. Wang, D. Huang, Q. Gu, Z. Zhao, and D. Xiang, Echoenabled tunable terahertz radiation generation with a lasermodulated relativistic electron beam, Phys. Rev. Accel. Beams 17, 090701 (2014).

[26] Z. Zhang, L. Yan, Y. Du, W. Huang, C. Tang, and Z. Huang, Generation of high-power, tunable terahertz radiation from laser interaction with a relativistic electron beam, Phys. Rev. Accel. Beams 20, 050701 (2017).

[27] T. Tanaka, Difference frequency generation in free electron lasers, Opt. Lett. 43, 4485 (2018). 